\title{
ARTICLE OPEN \\ Features of COPD patients by comparing CAT with mMRC: a retrospective, cross-sectional study
}

\author{
Wei-Chang Huang ${ }^{1,2}$, Ming-Feng $\mathrm{Wu}^{1,3}$, Hui-Chen $\mathrm{Chen}^{1}$, Jeng-Yuan Hsu ${ }^{4,5,6}$ and the TOLD Group ${ }^{7}$
}

\begin{abstract}
BACKGROUND: The group assignment of chronic obstructive pulmonary disease (COPD) may differ depending on whether the COPD assessment test (CAT) or modified Medical Research Council dyspnoea scale (mMRC) is used.

AIMS: This study intended to clarify how different patient characteristics influence the differences, to determine the relationships between CAT and MMRC and to characterise COPD patients by both CAT and MMRC.

METHODS: This was a retrospective, cross-sectional study. The data, collected by Taiwan Obstructive Lung Disease consortium, were managed and analysed.

RESULTS: Of the 757 participants, COPD group assignment was not identical as well as no substantial agreement presented when categorised based on the cut-point CAT score $\geqslant 10$ and each mMRC cut-point. In all, 38.2\% of participants had discordant group assignments together with a lower mean CAT score, less severe airway obstruction and less severe airflow limitation compared with those with concordant group assignments. In the discordant group, the CAT $\geqslant 10 / \mathrm{mMRC} 0-1$ subgroup had more wheezing than CAT $<10 / m M R C \geqslant 2$ subgroup. Only moderate correlations existed between CAT and mMRC. More-symptom groups and combined high-risk group had better correlations than less-symptom groups and combined low-risk group, respectively. A modest negative correlation existed between forced expiratory volume in $1 \mathrm{~s}$ percentage (FEV $\%$ ) predicted and CAT score and between $\mathrm{FEV}_{1} \%$ predicted and $\mathrm{mMRC}$ scale in parallel with a significant positive relationship existing between the CAT score and mMRC scale. Notably, a significant proportion of COPD patients with each scale of mMRC had health status impairment.

CONCLUSIONS: The Global initiative for Chronic Obstructive Lung Disease committee should redefine the applications of CAT and mMRC in the management of COPD.
\end{abstract}

npj Primary Care Respiratory Medicine (2015) 25, 15063; doi:10.1038/npjpcrm.2015.63; published online 5 November 2015

\section{INTRODUCTION}

The complexities of chronic obstructive pulmonary disease (COPD) require a comprehensive assessment for its management. The Global initiative for Chronic Obstructive Lung Disease (GOLD) committee has provided recommendations for the appropriate diagnosis and treatment of COPD. Emerging evidence indicates that the degree of airflow limitation was poorly predictive of dyspnoea and quality of life. ${ }^{1-3}$ Therefore, the GOLD committee in 2011 moved away from a linear, one-dimensional classification of severity groups, defined solely by degree of airflow limitation (forced expiratory volume in $1 \mathrm{~s}\left(\mathrm{FEV}_{1}\right)$ ), to a two-dimensional assessment that takes into account both exacerbation risk and symptom assessment. ${ }^{4}$

The exacerbation risk of COPD is determined by exacerbation history in the previous 1 year and spirometric classification of airflow limitation by the GOLD grade categorised by $\mathrm{FEV}_{1} \%$ predicted. This symptom is measured by either the COPD assessment test (CAT) or the modified Medical Research Council dyspnoea scale (mMRC) although the Clinical COPD Questionnaire was also proposed by GOLD 2013 revision. High risk includes GOLD 3 or 4 (severe or very severe) or a history of $\geqslant 2$ exacerbations in the previous year. The highest risk should be used if there is a discrepancy between the risk category as determined by the spirometric classification and that derived by the exacerbation history. Otherwise, low risk will be the case. The COPD patient's level of symptoms is classified into either less or more symptoms. A CAT score $\geqslant 10$ or $\mathrm{mMRC}$ scale $\geqslant 2$ indicates more symptoms, otherwise less symptoms will be the case. This combination of assessments classifies COPD patients into one of the four categories: A (low risk and less symptoms), B (low risk and more symptoms), $C$ (high risk and less symptoms), and D (high risk and more symptoms). The management strategy is thereby determined according to this classification.

The CAT comprises eight items relating to the severity of cough, sputum, dyspnoea, chest tightness, capacity for exercise and activities, confidence, sleep quality and energy levels, ${ }^{5}$ while the mMRC is a quantitative assessment tool only for breathlessness.

Although GOLD 2011 recommended a CAT score $\geqslant 10$ corresponding to a mMRC scale $\geqslant 2$ in symptom assessment for categorising COPD patients into less- or more-symptom groups, several studies have found that the group assignment of COPD produced by these cut-points was not identical. ${ }^{6-8}$

To understand the impacts of GOLD 2011 on COPD patients in Taiwan, the Taiwan Obstructive Lung Disease (TOLD) consortium was set up and is comprised of 12 teaching hospitals throughout Taiwan. We hypothesised that group assignment of COPD by the

\footnotetext{
${ }^{1}$ Division of Chest Medicine, Department of Internal Medicine, Taichung Veterans General Hospital, Taichung, Taiwan; ${ }^{2}$ Department of Medical Technology, Jen-Teh Junior College of Medicine, Nursing and Management, Miaoli, Taiwan; ${ }^{3}$ Department of Medical Laboratory Science and Biotechnology, Central Taiwan University of Science and Technology, Taichung, Taiwan; ${ }^{4}$ Division of Critical Care and Respiratory Therapy, Department of Internal Medicine, Taichung Veterans General Hospital, Taichung, Taiwan; ${ }^{5}$ School of Medicine, China Medical University, Taichung, Taiwan and ${ }^{6}$ School of Physical Therapy, Chung-Shan Medical University, Taichung, Taiwan.

Correspondence: J-Y Hsu (hsujy@vghtc.gov.tw)

${ }^{7}$ The TOLD Group is listed before references.

Received 27 April 2015; revised 10 August 2015; accepted 8 September 2015
} 
cut-point CAT score $\geqslant 10$ was not exactly the same with that by the cut-point $m M R C$ scale $\geqslant 2$. The aim of this study was to clarify how different patient characteristics influence the differences, to determine the relationships between CAT and mMRC and to characterise COPD patients by both CAT and MMRC, as implemented by the TOLD consortium.

\section{MATERIALS AND METHODS}

Study design and population

The data were from a large-scale, cross-sectional, multi-centre, observational, retrospective study of the TOLD consortium conducted between November 2012 and August 2013 in 12 teaching hospitals throughout Taiwan. The participating physicians, who were qualified pulmonologists and actively involved in COPD management, screened outpatients for study entry. Patients aged $\geqslant 40$ years with a confirmed diagnosis of COPD based on the GOLD 2011 recommendation and a spirometry within the previous 1 year before enrollment were invited to participate. Patients were excluded if they participated in interventional clinical trials in the previous 1 year, had a history of asthma or, for the purpose of this study, did not complete both the CAT and mMRC. The hospitals' individual Institutional Review Board and Ethics Committees approved this study (approval number: CE13164) and informed consent was obtained from all participants.

\section{Data collection}

At a single study visit, participating physicians completed a detailed patient record form, which included baseline characteristics, smoking history, presence of absence of wheezing on listening to the chest at the outpatient clinics in the previous 1 year, spirometry, CAT scores, mMRC scales, exacerbation history in the previous 1 year, comorbidities of interest, including cardiovascular diseases (e.g., ischaemic heart disease, congestive heart failure, hypertension and arrhythmia), chronic lung diseases (e.g., previous pulmonary tuberculosis, bronchiectasis and pneumoconiosis) and lung cancer, and COPD groups according to the GOLD 2011 recommendation for each participant from medical records. After that, the patient record forms were collected for further data management and analysis.

\section{Symptom assessment}

The CAT is a questionnaire that is designed to measure health status of COPD patients. Eight statements assess the best and worst case scenarios of cough, phlegm, chest tightness, breathlessness going up hills/stairs, activity limitations at home, confidence leaving home, sleep and energy. Each statement is scored from 0 to 5 (best to worst) giving a total score range from 0 to $40 .^{5}$ The $\mathrm{MMRC}$, which is a patient-reported ordinal-rating scale, comprises five statements that describe almost the entire range of respiratory disability from none (Grade 0 ) to almost complete incapacity (Grade 4). ${ }^{9}$ For the purpose of this study, symptom evaluation for a GOLD grouping (A-D) was carried out using both questionnaires in each participant.

\section{Exacerbation risk}

Spirometry was taken from the documented evidence within the previous 1 year and interpreted according to the American Thoracic Society statement. ${ }^{10}$ Positive bronchodilator test (BT) was defined as FEV ${ }_{1}$ or forced vital capacity (FVC) improvement from predose value by $\geqslant 12 \%$ and $\geqslant 200 \mathrm{ml}$. An acute exacerbation was defined as a worsening of symptoms that required antibiotics or systemic steroids, emergency room visits or hospitalisations. A history of $\geqslant 2$ exacerbation in the previous 1 year was termed as frequent exacerbation. A participant with frequent exacerbation was referred to as a frequent exacerbator; otherwise non-frequent exacerbator was the referent.

\section{COPD patient group}

Participants were classified into four groups- $A, B, C$ or D-by their COPD symptoms as determined by CAT or mMRC and exacerbation risks as determined by GOLD spirometric classification and the history of exacerbations in the preceding year according to the GOLD 2011. However, for the purpose of this study, each individual participant was assigned twice, one with a CAT score and the other with the MMRC scale.
Statistical analysis

All data were expressed as mean and s.d. for continuous variables or number (percentage) for categorical variables. Comparisons were conducted using the independent $t$-test for continuous variables and chi-square test for categorical variables. Pearson's correlation coefficient was applied to test the relationship between CAT scores and mMRC scales, between post-BT FEV ${ }_{1} \%$ predicted and the CAT score and between post-BT $\mathrm{FEV}_{1} \%$ predicted and the mMRC scale. Analysis of variance was applied to test the association between the CAT score and mMRC scale. The kappa coefficient was used to interpret the extent of agreement between the two respiratory questionnaires (CAT versus $\mathrm{mMRC}$ ), where kappa $<0$ indicates a less than chance agreement and kappa $=1$ indicates a perfect agreement. Statistical significance was set at $P<0.05$. Statistical analysis was performed using the SPSS version 18.0 (SPSS, Chicago, IL, USA).

\section{RESULTS}

This observational study was conducted in 12 teaching hospitals throughout Taiwan, where 1054 subjects who came to outpatient clinics for any reason and who had a diagnosis of COPD were enrolled. However, out of the 1054 subjects, 297 subjects without the completion of both questionnaires were further excluded. In the end, 757 subjects who fulfilled the inclusion and exclusion criteria were included in the final analysis.

Table 1 shows the baseline characteristics of the enrolled participants. The overall mean age was $72.2 \pm 9.4$ years and the majority of participants were male. Cigarette smoking was the leading cause of COPD in $92.1 \%$ (697/757) of participants. Interestingly, frequent exacerbation, wheezing and positive BT were present in $13.6,44.8$ and $30.5 \%$ of the enrolled subjects, respectively.

For the purpose of this study, we evaluated the GOLD groups twice for each participant, once using the CAT score and again using the mMRC scale. Table 2 indicates that COPD group assignment was not identical when categorised based on the cutpoint CAT score $\geqslant 10$ and each mMRC cut-point. Based on the cut-points CAT score $\geqslant 10$ and $\mathrm{mMRC}$ scale $\geqslant 2$ recommended by the GOLD 2011, classifying patients by CAT resulted in 30.6, 17.3, 22.2 and $29.6 \%$ of participants in groups $A, B, C$ and $D$, whereas by mMRC resulted in 22.2, 26.0, 1.9 and $35.9 \%$, respectively. The agreement of group assignment was evaluated between by the cut-point CAT score $\geqslant 10$ and by using different cut-points of the mMRC scale. The best agreement of group assignment emerged when the cut-point CAT score $\geqslant 10$ corresponded to the cut-point $m M R C \geqslant 3$ (kappa $=0.55, P=0.000)$, whereas the worst agreement emerged when the cut-point CAT score $\geqslant 10$ corresponded to the cut-point mMRC $\geqslant 1 \quad$ (kappa $=0.36$, $P=0.000$ ) (Table 2). For patients with COPD, in terms of the agreement of group assignment, either no substantial or almost perfect agreement (kappa $>0.6$ ) was found between the cut-point CAT score $\geqslant 10$ and each mMRC cut-point.

Concordance was defined as the COPD group being classified based on the CAT score that was consistent with that defined by the mMRC scale. Conversely, discordance was defined as the COPD group being classified based on the CAT score, which was inconsistent with that defined by the MMRC scale. Table 1 shows that $38.2 \%(289 / 757)$ of the enrolled participants had a discordance in group assignment. Compared with the concordant group, the discordant group had a lower mean CAT score (7.9 \pm 5.1 versus $12.2 \pm 7.8, P=0.000$ ), less severe airway obstruction $\left(\mathrm{FEV}_{1} / \mathrm{FVC}\right.$ of $56.1 \pm 8.9$ versus $\mathrm{FEV}_{1} / \mathrm{FVC}$ of $54.2 \pm 10.2, P=0.009$ ) and less severe airflow limitation ( $\mathrm{FEV}_{1} \%$ predicted of $56.9 \pm 20.7$ versus $\mathrm{FEV}_{1} \%$ predicted of $54.0 \pm 22.1, P=0.072$ ).

The discordance group was divided into two subgroups: CAT $\geqslant 10 / m M R C ~ 0-1$ and CAT $<10 / m M R C \geqslant 2$. In all, $28.7 \%$ $(83 / 289)$ and $71.3 \%(206 / 289)$ of participants with discordant group assignment had CAT $\geqslant 10 /$ mMRC $0-1$ and CAT $<10 /$ $m M R C \geqslant 2$, respectively (Table 3 ). Compared with the CAT $<10 /$ 
Table 1. Baseline characteristics of the enrolled participants $(n=757)$

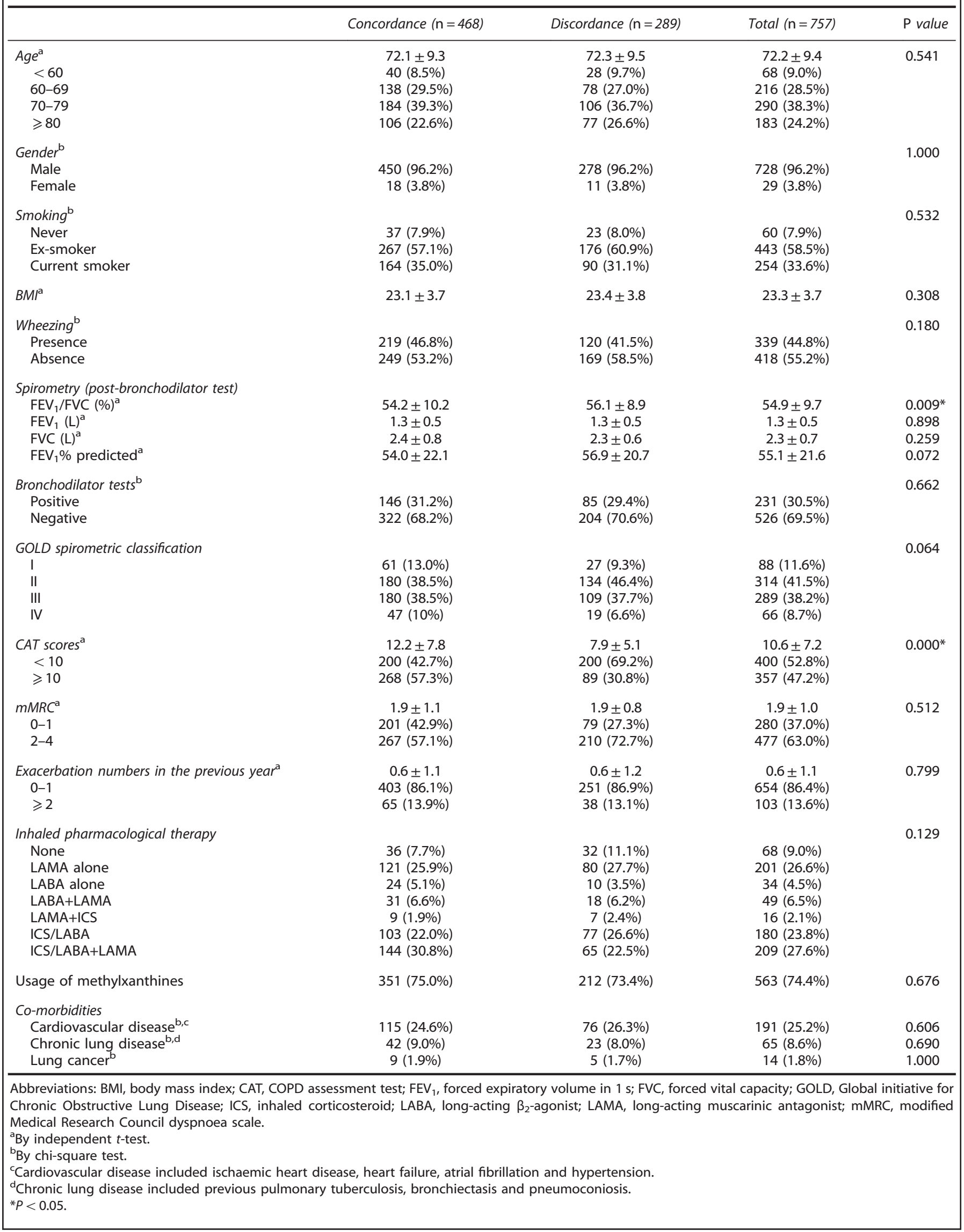


Table 2. Proportion of each COPD group (A-D) and agreement when categorised based on the cut-point CAT score $\geqslant 10$ and each mMRC cut-point

\begin{tabular}{lrrrrccc}
\hline & \multicolumn{4}{c}{ COPD group (\%) } & & Kappa value & P value \\
\cline { 2 - 5 } & \multicolumn{1}{c}{$A$} & \multicolumn{1}{c}{$B$} & \multicolumn{1}{c}{$C$} & \multicolumn{1}{c}{ D } & & \\
& & & & & \\
\hline CAT $\geqslant 10$ & 30.6 & 17.3 & 22.2 & 29.6 & Reference & \\
mMRC $\geqslant 1$ & 3.2 & 45.0 & 2.4 & 49.4 & 0.36 & $0.000^{*}$ \\
mMRC $\geqslant 2$ & 22.2 & 26.0 & 15.9 & 35.9 & 0.49 & $0.000^{*}$ \\
mMRC $\geqslant 3$ & 41.6 & 6.6 & 34.6 & 17.2 & 0.55 & $0.000^{*}$ \\
mMRC $=4$ & 48.0 & 0.3 & 47.8 & 0.4 & 0.40 & $0.000^{*}$ \\
\hline
\end{tabular}

Abbreviations: CAT, COPD assessment test; COPD, chronic obstructive pulmonary disease; mMRC, modified Medical Research Council dyspnoea scale. ${ }^{*} P<0.05$

$m M R C \geqslant 2$ subgroup, CAT $\geqslant 10 / m M R C 0-1$ subgroup had a higher percentage of presence of wheezing.

Table 4 shows that a moderate correlation existed between CAT and $\mathrm{mMRC}$ in the study population $\left(r=0.480, P=0.000^{*}\right)$. The regression model was CAT $=3.724+3.685 \times \mathrm{mMRC} \quad\left(R^{2}=0.230\right.$, $\mathrm{F}=225.440, P=0.000$ ). More-symptom groups (e.g., groups $B, D$ and $B+D$ ) had moderate correlations between these questionnaires whether classified by CAT or mMRC, whereas less symptom groups (e.g., groups A, C, A+C) had only weak-to-modest correlations except for the group $\mathrm{C}$ classified by mMRC. Moreover, the combined high-risk group (group $C+D$ ) had a moderate correlation between these questionnaires, whereas the combined low-risk group (group $\mathrm{A}+\mathrm{B}$ ) only had a modest correlation.

Figure 1 illustrates that a significant positive relationship existed between the CAT score and mMRC scale $(P=0.000)$. The mean CAT score $\geqslant 10$ occurred when the mMRC scale was $\geqslant 3$. Notably, a significant proportion of COPD patients with each scale of mMRC had health status impairment (CAT score $\geqslant 10$ ).

Figure 2 illustrates that the relationships between post-BT $\mathrm{FEV}_{1} \%$ predicted and the CAT score and between post-BT $\mathrm{FEV}_{1} \%$ predicted and the mMRC scale were only modestly negatively correlated.

Compared with those with CAT $<10$ and mMRC 0-1, COPD patients with CAT $\geqslant 10$ and $m M R C \geqslant 2$ were older in age and had a higher percentage of presence of wheezing, more severe airway obstruction, less $\mathrm{FEV}_{1}$ and FVC, more severe airflow limitation, parallel higher mMRC scales and higher CAT scores and more exacerbations, respectively (see Supplementary Tables S1 and S2 in the Supplementary Material).

\section{DISCUSSION}

Main findings

This study demonstrated that COPD group assignment was not identical when categorised based on the cut-point CAT score $\geqslant 10$ and each mMRC cut-point. Furthermore, no substantial or almost perfect agreement presented between the cut-point CAT score $\geqslant 10$ and each mMRC cut-point. In all, $38.2 \%$ of participants were found to have discordant group assignments together with a lower mean CAT score, less severe airway obstruction and less severe airflow limitation than those with concordant group assignment. In the discordant group, the CAT $\geqslant 10 / m M R C$ 0-1 subgroup had a higher percentage of presence of wheezing than the $C A T<10 / m M R C \geqslant 2$ subgroup. Neither perfect nor high correlation existed between CAT and mMRC even though the more-symptom groups and combined high-risk group had better correlations than the less-symptom groups and combined low-risk group, respectively. A modest negative correlation existed
Table 3. Detailed characteristics in COPD patients with discordant group assignments when divided into CAT $\geqslant 10 /$ mMRC $0-1$ and CAT $<10 /$ mMRC $\geqslant 2$ subgroups

\begin{tabular}{|c|c|c|c|}
\hline & $\begin{array}{c}C A T \geqslant 10 / m M R C \\
0-1(\mathrm{n}=83)\end{array}$ & $\begin{array}{c}C A T<10 / \\
m M R C \geqslant 2 \\
(\mathrm{n}=206)\end{array}$ & $\mathrm{P}$ value \\
\hline $\mathrm{Age}^{\mathrm{a}}$ & $71.7 \pm 10.2$ & $72.5 \pm 9.2$ & 0.485 \\
\hline Gender $^{\mathrm{b}}$ & & & 0.362 \\
\hline Male & 78 (94.0\%) & 200 (97.1\%) & \\
\hline Female & $5(6.0 \%)$ & $6(2.9 \%)$ & \\
\hline Smoking ${ }^{\mathrm{b}}$ & & & 0.132 \\
\hline Never & $8(9.6 \%)$ & $15(7.3 \%)$ & \\
\hline Ex-smoker & $43(51.8 \%)$ & $133(64.6 \%)$ & \\
\hline Current smoker & $32(38.6 \%)$ & $58(28.2 \%)$ & \\
\hline $\mathrm{BMI}^{\mathrm{a}}$ & $23.5 \pm 3.5$ & $23.4 \pm 3.9$ & 0.846 \\
\hline Wheezing $^{\mathrm{b}}$ & & & $0.008^{*}$ \\
\hline Presence & 45 (54.2\%) & 75 (36.4\%) & \\
\hline Absence & $38(45.8 \%)$ & $131(63.6 \%)$ & \\
\hline \multicolumn{4}{|c|}{ Spirometry (post- bronchodilator test) } \\
\hline $\mathrm{FEV}_{1} / \mathrm{FVC}(\%)^{\mathrm{a}}$ & $56.4 \pm 10.2$ & $55.9 \pm 8.3$ & 0.717 \\
\hline $\mathrm{FEV}_{1}(\mathrm{~L})^{\mathrm{a}}$ & $1.3 \pm 0.5$ & $1.3 \pm 0.5$ & 0.711 \\
\hline FVC $(L)^{a}$ & $2.3 \pm 0.6$ & $2.3 \pm 0.6$ & 0.713 \\
\hline $\mathrm{FEV}_{1} \%$ predicted $^{\mathrm{a}}$ & $55.7 \pm 21.9$ & $57.4 \pm 20.3$ & 0.535 \\
\hline Bronchodilator tests ${ }^{\mathrm{b}}$ & & & 1.000 \\
\hline Positive & $24(28.9 \%)$ & $61(29.6 \%)$ & \\
\hline Negative & $59(71.1 \%)$ & $145(70.4 \%)$ & \\
\hline CAT scores ${ }^{a}$ & $13.8 \pm 4.2$ & $5.5 \pm 3.0$ & $0.000^{*}$ \\
\hline $\mathrm{mMRC}^{\mathrm{a}}$ & $1.0 \pm 0.3$ & $2.2 \pm 0.5$ & $0.000^{*}$ \\
\hline $\begin{array}{l}\text { Exacerbation numbers in } \\
\text { the previous year }{ }^{\mathrm{a}}\end{array}$ & $0.7 \pm 1.1$ & $0.5 \pm 1.2$ & 0.345 \\
\hline \multicolumn{4}{|l|}{ Co-morbidities } \\
\hline $\begin{array}{l}\text { Cardiovascular } \\
\text { disease }^{b, c}\end{array}$ & $23(27.7 \%)$ & $53(25.7 \%)$ & 0.842 \\
\hline Chronic lung disease $^{b, d}$ & $4(4.8 \%)$ & 19 (9.2\%) & 0.241 \\
\hline Lung cancer ${ }^{\mathrm{b}}$ & $3(3.6 \%)$ & $2(1.0 \%)$ & 0.145 \\
\hline
\end{tabular}

Abbreviations: BMI, body mass index; CAT, COPD assessment test; COPD, chronic obstructive pulmonary disease; $\mathrm{FEV}_{1}$, forced expiratory volume in $1 \mathrm{~s}$; FVC, forced vital capacity; mMRC, modified Medical Research Council dyspnoea scale.

${ }^{\mathrm{a} B} \mathrm{By}$ independent $t$-test.

${ }^{\text {b }}$ By chi-square test.

'Cardiovascular disease included ischaemic heart disease, heart failure, atrial fibrillation and hypertension.

${ }^{d}$ Chronic lung disease included previous pulmonary tuberculosis, bronchiectasis and pneumoconiosis.

${ }^{*} P<0.05$.

between $\mathrm{FEV}_{1} \%$ predicted and CAT score and between $\mathrm{FEV}_{1} \%$ predicted and $\mathrm{mMRC}$ scale in parallel with a significant positive relationship existing between the CAT score and mMRC scale. Notably, a significant proportion of COPD patients with each scale of mMRC had health status impairment. COPD patients with more advanced age, wheezing, worse spirometric parameters, including more severe airway obstruction, less $\mathrm{FEV}_{1}$ and FVC and more severe airflow limitation, and more exacerbations were associated with a worse health status and respiratory capacity.

Interpretation of findings in relation to previously published work Consonant with the present study, several previous studies found that the group assignment of patients with COPD using the symptom assessment methods (CAT or mMRC) proposed by GOLD 
Table 4. Correlations between CAT and mMRC in each COPD group (A-D) and combined COPD groups when categorised based on GOLD 2011

\begin{tabular}{|c|c|c|c|c|}
\hline & \multicolumn{2}{|c|}{ Assignment by CAT } & \multicolumn{2}{|c|}{ Assignment by $m M R C$} \\
\hline & Number (\%) & $\begin{array}{l}\text { Pearson's } \\
\text { correlation }\end{array}$ & Number (\%) & $\begin{array}{l}\text { Pearson's } \\
\text { correlation }\end{array}$ \\
\hline \multicolumn{5}{|c|}{ Individual COPD group } \\
\hline A & $232(30.6)$ & $\begin{array}{l}r=0.010 \\
P=0.879\end{array}$ & $168(22.2)$ & $\begin{array}{c}r=0.189 \\
P=0.014^{*}\end{array}$ \\
\hline B & $133(17.6)$ & $\begin{aligned} r & =0.401 \\
P & =0.000^{*}\end{aligned}$ & $197(26.0)$ & $\begin{array}{l}r=0.431 \\
P=0.000^{*}\end{array}$ \\
\hline C & $168(22.2)$ & $\begin{array}{l}r=-0.060 \\
P=0.437\end{array}$ & $120(15.9)$ & $\begin{array}{l}r=0.465 \\
P=0.000^{*}\end{array}$ \\
\hline D & $224(29.6)$ & $\begin{array}{l}r=0.462 \\
P=0.000^{*}\end{array}$ & $272(35.9)$ & $\begin{aligned} r & =0.415 \\
P & =0.000^{*}\end{aligned}$ \\
\hline \multicolumn{5}{|c|}{ Combined COPD group } \\
\hline$A+C$ & $400(52.8)$ & $\begin{array}{l}r=-0.018 \\
P=0.713\end{array}$ & $288(38.0)$ & $\begin{aligned} r & =0.357 \\
P & =0.000^{*}\end{aligned}$ \\
\hline$B+D$ & $357(47.2)$ & $\begin{aligned} r & =0.460 \\
P & =0.000^{*}\end{aligned}$ & $469(62.0)$ & $\begin{array}{l}r=0.446 \\
P=0.000^{*}\end{array}$ \\
\hline$A+B$ & \multirow{2}{*}{\multicolumn{4}{|c|}{$\begin{array}{l}365(48.2), r=0.391, P=0.000^{*} \\
392(51.8), r=0.480, P=0.000^{*}\end{array}$}} \\
\hline$C+D$ & & & & \\
\hline All & \multicolumn{4}{|c|}{$757(100.0), r=0.480, P=0.000^{*}$} \\
\hline \multicolumn{5}{|c|}{$\begin{array}{l}\text { Abbreviations: CAT, COPD assessment test; COPD, chronic obstructive } \\
\text { pulmonary disease; GOLD, Global initiative for Chronic Obstructive Lung } \\
\text { Disease; mMRC, modified Medical Research Council dyspnoea scale. } \\
{ }^{*} P<0.05 \text {. }\end{array}$} \\
\hline
\end{tabular}

2011 was not consistent, $8,11,12$ which may be because the mMRC only assesses disability owing to breathlessness, whereas the CAT has a broader coverage of the impact of COPD on the patient's daily life. By using these two questionnaires, two previous studies showed that $53.7 \%(890 / 1659)$ and $27.2 \%$ (77/283) of enrolled participants had discordant COPD group assignments, respectively; ${ }^{8,11}$ and the present study revealed a discordance rate of $38.2 \%(289 / 757)$. Interestingly, we found that the discordance group had a lower CAT score, less severe airway obstruction and less severe airflow limitation. Furthermore, wheezing was associated with the CAT $\geqslant 10 /$ mMRC $0-1$ discordant subgroup. Although GOLD 2011 recommended that it is not necessary to use more than one symptom assessment questionnaire, these findings suggest that both simple tools, CAT and mMRC, ${ }^{5,13}$ should be assessed simultaneously for COPD patients with a lower CAT score, less severe airway obstruction and less severe airflow limitation in order to classify patients into optimal COPD groups and optimise COPD management. For COPD patients with inconsistent group assignment by using these two questionnaires, the presence of wheezing may imply not a worse respiratory capacity but a worse health status.

We found that there was a significant positive relationship between the CAT score and MMRC scale even though only a moderate correlation existed between these two questionnaires. We also found that a significant proportion of COPD patients with each scale of mMRC had health status impairment (CAT score $\geqslant 10$ ). These findings were similar with those reported by Jones et al. ${ }^{12}$ and shows that, notwithstanding the existence of a positive correlation, the CAT score $\geqslant 10$ serving as an equivalence to an mMRC score $\geqslant 2$ as recommended by GOLD 2011 may not be applicable to all COPD patients. In other words, COPD patients categorised as having less symptoms using the cut-point $m M R C \geqslant 2$ (the GOLD 2011 recommendation) may have a worse health status and vice versa.

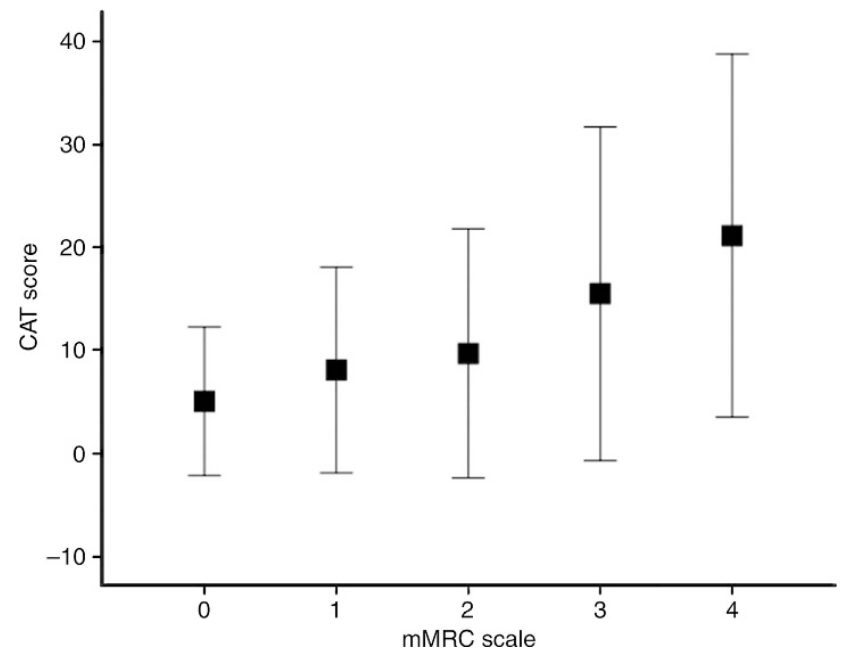

Figure 1. The relationship between the CAT score and mMRC scale for the study COPD population. Data were presented as mean \pm 2 s.d. $P=0.000$ for the one-way ANOVA of the association between the CAT score and mMRC scale.

We observed that a lower mean CAT score for each mMRC scale compared with findings reported in one previous study conducted in a western country. ${ }^{12}$ Although the baseline characteristics of enrolled COPD patients in the present study were similar to several published studies, the distribution of COPD groups was different from one study to another. ${ }^{7,11,12}$ As a result, different geographical and sociocultural environments may have different impacts on symptom assessment and COPD categorisation.

Regarding the cut-points CAT score $\geqslant 10$ and mMRC scale $\geqslant 2$ that were recommended by GOLD 2011, our study reiterated the findings of several previous studies that reported that these two questionnaires had only moderate agreement in the categorisation of COPD groups. ${ }^{711,12,14}$ In contrast, one previous large-scale cohort study found that the cut-points CAT score $\geqslant 10$ and mMRC scale $\geqslant 1$ were approximately equivalent in determining COPD groups with substantial agreement. ${ }^{12}$ Although the cut-points CAT score $\geqslant 10$ and mMRC scale $\geqslant 3$ had the best agreement (kappa $=0.55, P=0.000$ ) in the present study, it was the case that only moderate agreement was observed. Our findings suggest that there was no optimal cut-point for $\mathrm{mMRC}$ to correspond to the cut-point CAT score $\geqslant 10$, which differed from the results reported in the previously mentioned large-scale study. ${ }^{12}$ This can be explained by the different study designs between these two studies. The large-scale cohort study was conducted in a primary care setting and the number of exacerbations was recorded only in the previous 6 months; also, spirometry was not performed in a standardised way across the study institutes. In contrast, our study design, as mentioned in the paragraph under the heading 'Strengths and limitations of this study', was more rigorous and therefore better represented the true distribution of COPD groups and agreement of group assignments between the CAT score and the mMRC scale of COPD patients.

Although COPD patients with worse spirometric parameters were associated with a worse health status $($ CAT $\geqslant 10)$ and respiratory capacity $(\mathrm{mMRC} \geqslant 2)$, along with our findings, several studies found that respiratory disease questionnaires, including St George's Respiratory Questionnaire total scores, CAT scores and mMRC scales, had only a weak negative correlation with $\mathrm{FEV}_{1} \%$ predicted. ${ }^{15,16}$ This indicates that, with any given pulmonary function reserve, COPD patients may range from having relatively well-preserved to very poor health status and from no respiratory disability to almost complete respiratory incapacity. 

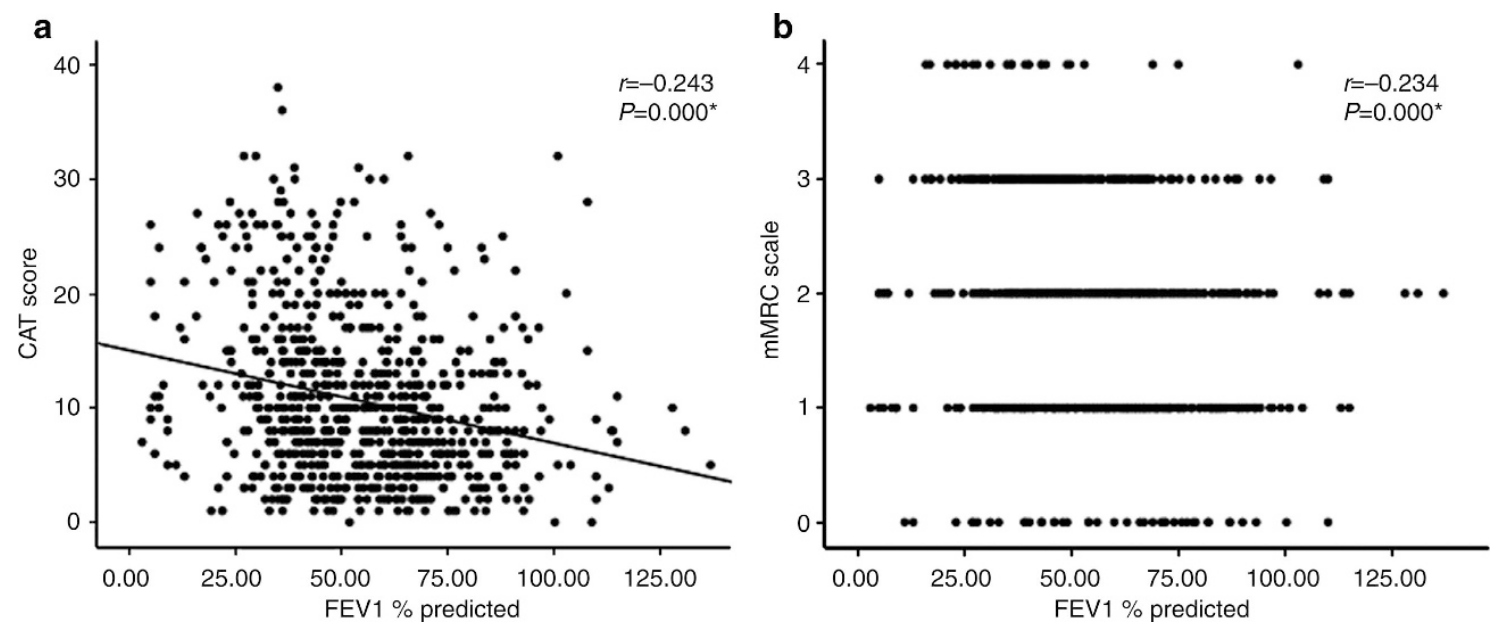

Figure 2. Relationships (a) between post-bronchodilator test forced expiratory volume in $1 \mathrm{~s} \%$ predicted (post-BT FEV $1 \%$ predicted) and the CAT score and (b) between post-BT $\mathrm{FEV}_{1} \%$ predicted and the mMRC scale. ${ }^{*} P<0.05$.

One previous global trial found that $13.6 \%$ of moderate-to-verysevere COPD patients were frequent exacerbators who were closely associated with exacerbation-related hospitalisations and poorer survival. ${ }^{17}$ We found that $13.6 \%$ of mild-to-very-severe COPD patients had frequent exacerbation. Furthermore, more exacerbations in the previous year were associated with a worse health status $(C A T \geqslant 10)$ and respiratory capacity $(m M R C \geqslant 2)$. This indicates that, compared with non-frequent exacerbators, frequent exacerbators had a worse health status and respiratory capacity, which may explain why frequent exacerbators tended to have exacerbation-related hospitalisations and poorer survival. ${ }^{17}$

One previous study indicated that only $2.6 \%(6 / 230)$ of nonsmoker COPD patients and 1.9\% (7/375) of smoker COPD patients had a history of wheezing at the outpatient clinics. ${ }^{18}$ We found that $44.8 \%$ of stable COPD patients had wheezing and were associated with a worse health status (CAT $\geqslant 10)$ and respiratory capacity $(m M R C \geqslant 2)$, compared with those without wheezing. However, the implications remain unclear regarding the association of wheezing and important clinical outcomes (such as the response to inhaled corticosteroids or long-acting $\beta_{2}$-agonist), risks of exacerbation and hospitalisation and mortality in stable COPD patients. Further research should thus explore the clinical relevance of wheezing in stable COPD patients.

Unlike the Understanding Potential Long-term Impacts on Function with Tiotropium study and the report by Zhang et al. ${ }^{18}$ showing a bronchodilator reversibility in $53.9 \%$ and $8.4 \%$ of COPD patients, respectively, we found that $30.5 \%$ of COPD patients exhibited airway reversibility. ${ }^{18,19}$ Along with our findings, other evidence suggests that reversibility results cannot predict a COPD patient's quality of life, respiratory capacity or long-term response to maintenance bronchodilator treatment. ${ }^{20,21}$

Similar to the results in a cross-sectional study conducted in Europe, ${ }^{15}$ we found that there were no associations between any COPD-related co-morbidity and health status and between any COPD-related co-morbidity and respiratory capacity. These findings indicate that the quality of life and respiratory capacity of COPD patients were not affected by any COPD-related co-morbidity. However, COPD patients with simultaneous $\geqslant 3$ co-morbidities would have a higher CAT score. ${ }^{15}$

Strengths and limitations of this study

This study was implemented by qualified pulmonologists who were actively involved in COPD management in a referral hospital setting, and in order to comply with the GOLD 2011 recommendation, the number of exacerbations was recorded in the previous
12 months; we also performed spirometry according to the American Thoracic Society statement in all study institutes. We believe that our study design was rigorous and therefore represented the true distribution of COPD groups and agreement of group assignments between the CAT score and the mMRC scale of COPD patients.

There were several limitations in our study. First, instead of recording all COPD-related co-morbidities, we only kept a record of the co-morbidities of interest, including cardiovascular diseases, chronic lung diseases and lung cancer. As a result, the effect of COPD-related co-morbidities on health status and respiratory capacity could not be evaluated comprehensively. Second, the participants were not sampled randomly. COPD patients with worse health status and respiratory capacity (e.g., CAT score $\geqslant 30$ and $\mathrm{mMRC}$ scale $=4$ ) had less will to participate in the study. Hence, underestimations of the overall CAT scores and mMRC scales, the proportion of more-symptom group and the proportion of concordant group assignment may exist. Third, as few as $7.9 \%$ (60/757) of participants were non-smoker COPD patients in the present study. Therefore, our findings may not be applicable to patients with COPD who are associated with risk factors other than cigarette smoking.

Implications for future research, policy and practice

In contrast to the GOLD 2011 recommendation that either the CAT score or mMRC scale can be the symptom assessment questionnaire, we have shown evidence that both the CAT score and $\mathrm{mMRC}$ scale should be evaluated simultaneously for COPD patients with a lower CAT score, less severe airway obstruction and less severe airflow limitation. Although GOLD 2011 recommended that CAT is preferred as it provides a more comprehensive assessment of the symptomatic impact on patients with COPD, how to categorise COPD patients with inconsistent group assignment based on these two respiratory questionnaires to optimise COPD management as well as how to choose more suitable questionnaires for special types of COPD patients need to be validated further in future studies.

\section{Conclusions}

In contrast to the GOLD 2011 recommendation, COPD patients with a lower CAT score, less severe airway obstruction and less severe airflow limitation were associated with discordant group assignments and should evaluate their symptoms with both CAT and mMRC simultaneously. We did not find any optimal cut-point for $\mathrm{mMRC}$ to correspond to the cut-point CAT score $\geqslant 10$. The 
GOLD committee should redefine the applications of CAT and mMRC in the management of COPD.

\section{ACKNOWLEDGEMENTS}

We thank the collaborators and members of the TOLD study group.

\section{THE TOLD STUDY GROUP}

The principal investigators of the TOLD study group include Ying-Huang Tsai ${ }^{8}$ Chi-Wei Tao ${ }^{9}$, Shih-Lung Cheng ${ }^{10}$, Chao-Hsien Lee ${ }^{11}$, Ping-Hung Kuo ${ }^{12}$, Yao-Kuang $\mathrm{Wu}^{13}$, Ning-Hung Chen ${ }^{14}$, Wu-Huei $\mathrm{Hsu}^{15}$, Jeng-Yuan $\mathrm{Hsu}^{16}$, Ming-Shian Lin ${ }^{17}$, Chin-Chou Wang ${ }^{18}$ and Yu-Feng Wei ${ }^{19}$

${ }^{8}$ Chang Gung Memorial Hospital, Chiayi, Taiwan;

${ }^{9} \mathrm{Cheng}-\mathrm{H}$ sin General Hospital, Taipei, Taiwan;

${ }^{10}$ Far Eastern Memorial Hospital, Taipei, Taiwan;

${ }^{11}$ Mackay Memorial Hospital, Taipei, Taiwan;

${ }^{12}$ National Taiwan University Hospital, Taipei, Taiwan;

${ }^{13}$ Taipei Tzu Chi Hospital, Taipei, Taiwan;

${ }^{14}$ Chang Gung Memorial Hospital, Linkou, Taiwan;

${ }^{15}$ China Medical University Hospital, Taichung, Taiwan;

${ }^{16}$ Taichung Veterans General Hospital, Taichung, Taiwan;

${ }^{17}$ Chia-Yi Christian Hospital, Chiayi, Taiwan;

${ }^{18}$ Chang Gung Memorial Hospital, Kaohsiung, Taiwan;

${ }^{19}$ E-DA Hospital, Kaohsiung, Taiwan.

\section{CONTRIBUTIONS}

Jeng-Yuan Hsu and Wei-Chang Huang designed the study. All the authors performed the study. Wei-Chang Huang, Hui-Chen Chen and Jeng-Yuan Hsu the collected data. Wei-Chang Huang, Ming-Feng Wu and Jeng-Yuan Hsu analysed the data. Wei-Chang Huang and Jeng-Yuan Hsu wrote the paper.

\section{COMPETING INTERESTS}

Wei-Chang Huang and Jeng-Yuan Hsu declared receiving lecture fees from Novartis Pharmaceuticals. The remaining authors declare no conflict of interest.

\section{FUNDING}

The TOLD study received financial support from Novartis Pharmaceuticals. The editorial assistance was provided by Asia Training Solutions, and the data management and statistical analysis were supported by CRO service division of Formosa Biomedical Technology.

\section{REFERENCES}

1 Garcia-Aymerich J, Serra Pons I, Mannino DM, Maas AK, Miller DP, Davis KJ. Lung function impairment, COPD hospitalisations and subsequent mortality. Thorax 2011; 66: 585-590.

2 Hurst JR, Vestbo J, Anzueto A, Locantore N, Müllerova $\mathrm{H}$, Tal-Singer $\mathrm{R}$ et al. Evaluation of COPD Longitudinally to Identify Predictive Surrogate Endpoints (ECLIPSE) Investigators. Susceptibility to exacerbation in chronic obstructive pulmonary disease. N Engl J Med 2010; 363: 1128-1138.

3 Weatherall M, Marsh S, Shirtcliffe P, Williams M, Travers J, Beasley R. Quality of life measured by the St George's Respiratory Questionnaire and spirometry. Eur Respir J 2009; 33: 1025-1030.
4 Global Initiative for Chronic Obstructive Lung Disease (GOLD). Global strategy for the diagnosis, management, and prevention of chronic obstructive pulmonary disease: revised 2011. Available from http://www.goldcopd.org/uploads/users/ files/GOLD_Report_2011_Jan21.pdf.

5 Jones PW, Harding G, Berry P, Wiklund I, Chen WH, Kline Leidy N. Development and first validation of the COPD Assessment Test. Eur Respir J 2009; 34: 648-654.

6 Han MK, Muellerova H, Curran-Everett D, Dransfield MT, Washko GR, Regan EA et al. GOLD 2011 disease severity classification in COPDGene: a prospective cohort study. Lancet Respir Med 2013; 1: 43-50.

$7 \mathrm{Kim}$ S, Oh J, Kim Yl, Ban HJ, Kwon YS, Oh IJ et al. Differences in classification of COPD group using COPD assessment test (CAT) or modified Medical Research Council (mMRC) dyspnea scores: a cross-sectional analysis. BMC Pulm Med 2013; 13: 35.

8 Price DB, Baker CL, Zou KH, Higgins VS, Bailey JT, Pike JS. Real-world characterization and differentiation of the Global Initiative for Chronic Obstructive Lung Disease strategy classification. Int J Chron Obstruct Pulmon Dis 2014; 9: 551-561.

9 Mahler DA, Wells CK. Evaluation of clinical methods for rating dyspnea. Chest 1988; 93: 580-586.

10 American Thoracic Society Statement. Lung function testing: selection of reference values and interpretative strategies. Am Rev Respir Dis 1991; 144: 1202-1218.

11 Rieger-Reyes C, García-Tirado FJ, Rubio-Galán FJ, Marín-Trigo JM. Classification of chronic obstructive pulmonary disease severity according to the new Global Initiative for Chronic Obstructive Lung Disease 2011 guidelines: COPD assessment test versus modified Medical Research Council scale. Arch Bronconeumol 2014; 50: 129-134.

12 Jones PW, Adamek L, Nadeau G, Banik N. Comparisons of health status scores with MRC grades in COPD: implications for the GOLD 2011 classification. Eur Respir J 2013; 42: 647-654.

13 Jones $P$, Harding G, Wiklund I, Berry P, Leidy N. Improving the process and outcome of care in COPD: development of a standardised assessment tool. Prim Care Respir J 2009; 18: 208-215.

14 Holt S, Sheahan D, Helm C, Tofield C, Corin A, Kocks JW. Little agreement in GOLD category using CAT and mMRC in 450 primary care COPD patients in New Zealand. NPJ Prim Care Respir Med 2014; 24: 14025.

15 Jones PW, Brusselle G, Dal Negro RW, Ferrer M, Kardos P, Levy ML et al. Properties of the COPD assessment test in a cross-sectional European study. Eur Respir J 2011; 38: 29-35.

16 Jones PW. Health status and the spiral of decline. COPD 2009; 6: 59-63.

17 Beeh KM, Glaab T, Stowasser S, Schmidt H, Fabbri LM, Rabe KF et al. Characterisation of exacerbation risk and exacerbator phenotypes in the POET-COPD trial. Respir Res 2013; 14: 116.

18 Zhang J, Lin XF, Bai CX. Comparison of clinical features between non-smokers with COPD and smokers with COPD: a retrospective observational study. Int $J$ Chron Obstruct Pulmon Dis 2014; 9: 57-63.

19 Tashkin DP, Celli B, Decramer M, Liu D, Burkhart D, Cassino C et al. Bronchodilator responsiveness in patients with COPD. Eur Respir J 2008; 31: 742-750.

20 Mahler DA, Donohue JF, Barbee RA, Goldman MD, Gross NJ, Wisniewski ME et al. Efficacy of salmeterol xinafoate in the treatment of COPD. Chest 1999; 115: 957-965.

21 Tashkin D, Kesten S. Long-term treatment benefits with tiotropium in COPD patients with and without short-term bronchodilator responses. Chest 2003; 123: 1441-1449.

\footnotetext{
(c) This work is licensed under a Creative Commons Attribution 4.0 International License. The images or other third party material in this article are included in the article's Creative Commons license, unless indicated otherwise in the credit line; if the material is not included under the Creative Commons license, users will need to obtain permission from the license holder to reproduce the material. To view a copy of this license, visit http://creativecommons.org/licenses/ by/4.0/
}

Supplementary Information accompanies the paper on the npj Primary Care Respiratory Medicine website (http://www.nature.com/npjpcrm) 